\title{
Sex-related differences in predicting choledocholithiasis using current American Society of Gastrointestinal Endoscopy risk criteria
}

\section{Ankit Chhoda ${ }^{a}$, Deepanshu Jain ${ }^{b}$, Shashideep Singhalc}

Yale-Waterbury Internal Medicine Program, Yale School of Medicine, Waterbury Connecticut; Albert Einstein Medical Center, Philadelphia; University of Texas Health Science Center at Houston, Houston, Texas, USA

\section{Abstract}

${ }^{a}$ Department of Internal Medicine, Yale-Waterbury Internal Medicine Program, Yale School of Medicine, Waterbury Connecticut (Ankit Chhoda); ${ }^{b}$ Division of Gastroenterology and Hepatology, Department of Internal Medicine, Albert Einstein Medical Center, Philadelphia, PA (Deepanshu Jain); 'Division of Gastroenterology, Hepatology and Nutrition, University of Texas Health Science Center at Houston, Houston, Texas (Shashideep Singhal), USA

Conflict of Interest: None

Correspondence to: Shashideep Singhal, MD, Division of Gastroenterology, Hepatology and Nutrition, University of Texas Health Science Center at Houston, 6431 Fannin, MSB 4.234, Houston, Texas, USA 77030, e-mail: sdsinghal@gmail.com

Received 31 July 2017; accepted 4 September 2017; published online 10 October 2017

DOI: https://doi.org/10.20524/aog.2017.0198

Background American Society of Gastrointestinal Endoscopy (ASGE) criteria is widely used to predict probability of choledocholithiasis among patients with symptomatic gallstone disease but does not take sex into consideration.

Methods The cohort study included patients who underwent either endoscopic retrograde cholangiopancreatography or intraoperative cholangiography for suspected choledocholithiasis at our medical center. Clinical, laboratory and radiological data were collected for each patient and used to stratify them based on the ASGE risk criteria for choledocholithiasis.

Results A total of 646 patients composed the final study cohort. The composite incidence rate of choledocholithiasis for male and female groups was $47.5 \%$ and $46.2 \%$ respectively. Total bilirubin $(>4 \mathrm{mg} / \mathrm{dL}$ ), cholangitis, abnormal liver function tests (transaminitis), dilation of the common bile duct $(>6 \mathrm{~mm})$ on ultrasound examination, and biliary pancreatitis were individually associated with choledocholithiasis in $73.5 \%, 68.4 \%, 61.1 \%, 60.0 \%$, and $51.7 \%$ of males, respectively. Total bilirubin $>4 \mathrm{mg} / \mathrm{dL}$ and $1.8-4 \mathrm{mg} / \mathrm{dL}$, cholangitis, and transaminitis were individually associated with choledocholithiasis in $82.6 \%, 64.0 \%, 58.2 \%$, and $50.0 \%$ of females, respectively. The distribution in probability group/incidence of choledocholithiasis was as follows: for males, low probability $3.3 \% / 0 \%$, intermediate probability $56.7 \% / 33.8 \%$ ), high probability $40 \% / 77.8 \%$; and for females, low probability $5.3 \% / 14.3 \%$, intermediate probability $70.2 \% / 39.6 \%$, high probability $24.5 \% / 72.1 \%$.

Conclusions The composite incidence for choledocholithiasis was similar across male and female patients. A significantly higher proportion of females compared to males were in the intermediate probability group. Better sex stratification can help improve the positive and negative predictive values of ASGE risk stratification criteria. Improve patient outcomes and reduce associated healthcare cost.

Keywords Gallstones, choledocholithiasis, sex distribution

Ann Gastroenterol 2018; 31 (1): 1-6SS 


\section{Introduction}

Gallstone disease is a widespread disorder affecting 20 million Americans. It is diagnosed by demonstration of a stone in the gallbladder using transabdominal ultrasound (US) or, based on a history of cholecystectomy [1]. Female sex has been identified as one of the major risk factor for gallstone disease [2,3]. The increased incidence, however, decreases by the fifth decade and the incidence in both sexes becomes comparable [4].

Along with cholecystitis, cholangitis, biliary pancreatitis and choledocholithiasis are complications of gallstone disease, caused either by migration of the stone from the gallbladder, or by intraductal precipitation due to bile stasis, biliary diverticulum or a large bile duct. It has been estimated that the frequency of stones in the common bile duct (CBD) during cholecystectomy ranges from 5-20\%; however, the exact prevalence remains unknown [5-8].

The criteria of the American Society of Gastrointestinal Endoscopy (ASGE) are widely used for the prediction of choledocholithiasis and management based on risk stratification (Table 1) [9]. The aim of our study was to determine sex-related differences in the prediction of choledocholithiasis in patients with symptomatic gallstones.

\section{Patients and methods}

\section{Study location}

University hospital and a community hospital under the University of Texas Health Science Center, Houston.

Table 1 ASGE recommended criteria for predicting choledocholithiasis

Predictors of choledocholithiasis

\begin{tabular}{|c|c|}
\hline \multicolumn{2}{|l|}{$\begin{array}{l}\text { Very strong } \\
\text { - CBD stone on transabdominal US } \\
\text { - Clinical ascending cholangitis } \\
\text { - Bilirubin }>4 \mathrm{mg} / \mathrm{dL}\end{array}$} \\
\hline $\begin{array}{l}\text { Strong } \\
\text { - Dilated CBD on US ( } 6 \mathrm{~mm} \text { with gallbl } \\
\text { - Bilirubin level } 1.8-4 \mathrm{mg} / \mathrm{dL}\end{array}$ & situ) \\
\hline $\begin{array}{l}\text { Moderate } \\
\text { - Abnormal liver biochemical test other } \\
\text { - Age }>55 \text { years } \\
\text { - Clinical gallstone pancreatitis }\end{array}$ & irubin \\
\hline $\begin{array}{l}\text { Assigning a likelihood of choledocholithi } \\
\text { predictors } \\
\text { - Presence of any very strong predictor } \\
\text { - Presence of both strong predictors } \\
\text { - No predictors present } \\
\text { - All other patients }\end{array}$ & $\begin{array}{l}\mathrm{d} \text { on clinical } \\
\text { High } \\
\text { High } \\
\text { Low } \\
\text { Intermediate }\end{array}$ \\
\hline
\end{tabular}

ASGE, American Society of Gastrointestinal Endoscopy; CBD, common bile duct; US, ultrasound examination

\section{Study duration}

The study covered the 3 years from November 1, 2012 to August 1, 2015.

\section{Inclusion criteria}

1. Patients undergoing endoscopic retrograde cholangiopancreatography (ERCP) for suspected choledocholithiasis: high probability based on ASGE criteria, or patients with intermediate probability where immediate cholecystectomy was not feasible due to their medical condition.

2. Patients who underwent intraoperative cholangiography (IOC) at the time of cholecystectomy: low/intermediate probability for choledocholithiasis. All patients with a positive IOC subsequently underwent ERCP.

IOC during cholecystectomy is a reliable modality recommended in ASGE guidelines for the evaluation of choledocholithiasis [9]. At our institution, most patients with intermediate or low probability for choledocholithiasis underwent IOC routinely during cholecystectomy. In our experience, this approach can save time and avoid additional investigations, such as endoscopic US or magnetic resonance cholangiopancreatography. Only a small proportion of patients with intermediate probability who were at high risk for cholecystectomy underwent ERCP as a temporary measure, while awaiting medical optimization for cholecystectomy. Fig. 1 illustrates the numbers of patients in each category.

\section{Exclusion criteria}

Patients who were being followed up for incomplete stone clearance from previous ERCP and stent replacement were excluded.

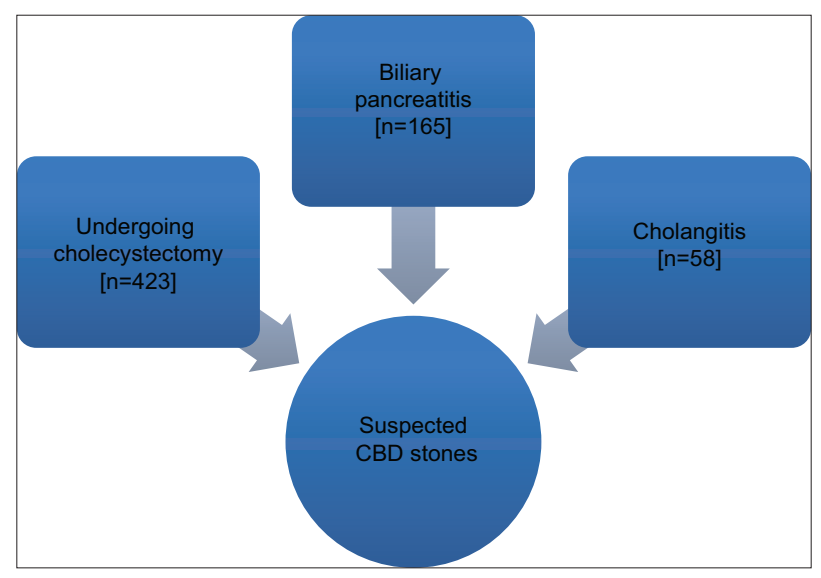

Figure 1 Study flow diagram $C B D$, common bile duct 


\section{Data collection}

Clinical data at presentation (age, sex, symptomatology, laboratory and imaging parameters) and procedural (IOC and ERCP) details with complications were collected. The preERCP imaging, which included abdominal US, was reviewed to collect data regarding CBD stone visualization with acoustic shadowing and CBD diameter.

\section{Methodology}

Our study was retrospective and observational in nature. After the relevant data had been collected, the study cohort was divided into two groups based on sex. Each group was further stratified into low-, intermediate- or high-risk category for choledocholithiasis based on the ASGE risk factors. The true incidence rate for choledocholithiasis for each category in our study was then compared with the predicted risk to ascertain any clinically significant differences.

\section{Statistical analysis}

Statistical analysis was performed using SPSS version 23.0. Quantitative data were represented as mean \pm standard deviation and qualitative data as numbers and percentages. Statistical differences were investigated using the $t$-test for continuous variables and the chi-square test for categorical variables. Any difference or association between two variables with a P-value $<0.05$ was considered to be statistically significant. Beta error was set at $20 \%$ and hence power at $80 \%$.

\section{Variable definition}

Ascending cholangitis was defined as fever (temperature $>37.8^{\circ} \mathrm{C}$ ) with abdominal pain and jaundice. Biliary pancreatitis was defined as elevated lipase levels (three times the institutional cutoff) in the absence of other risk factors, such as alcohol ingestion or medications associated with pancreatitis.

\section{ERCP procedure}

The ERCP was performed using Olympus TJF 160 VR or TJF 145 side-viewing endoscopes. All patients provided written consent to undergo ERCP and were informed of the risks and benefits of the procedure. Patients underwent propofol sedation assisted by an anesthesiologist or conscious sedation from midazolam or ketamine administered by the endoscopist. An initial cholangiogram was performed to determine the maximum diameter of the $\mathrm{CBD}$ and the number and size of the stones. The patients underwent endoscopic sphincterotomy over a guidewire. The stones were retrieved via a balloon catheter or a Dormia basket. The bile duct clearance was documented with a balloon catheter occlusion cholangiogram at the end of the procedure. A biliary stent was placed for residual CBD stone and follow-up ERCP was scheduled. The patients were followed up for post-procedural complications on the following day.

\section{Results}

A total of 646 patients satisfied the inclusion and exclusion criteria. The patients in our study were predominantly female $(n=526)$ rather than male $(n=120)$. The median age was significantly less for males (36.3 years) than for females (49 years) $(\mathrm{P}<0.0001)$. ASGE risk factors among male and female patients were also compared for the incidence of CBD stones (Table 2). None of the risk factors showed a significant sex-related difference in the incidence of CBD. Logistic univariate analysis was performed to determine the association of the risk factors with choledocholithiasis for both sexes (Supplemental Tables 1 \& 2). Apart from abnormal liver function tests (LFTs) among males and dilated CBD

Table 2 Distribution of ASGE risk factors across male and female patients

\begin{tabular}{|c|c|c|c|c|c|c|}
\hline \multirow[t]{2}{*}{ ASGE risk factors } & \multicolumn{2}{|c|}{ Male patients $(n=120)$} & \multicolumn{2}{|c|}{ Female patients $(n=526)$} & \multirow{2}{*}{$\begin{array}{c}\text { P-value } \\
\text { risk factors }\end{array}$} & \multirow{2}{*}{$\begin{array}{c}\text { P-value } \\
\text { CBD stone incidence }\end{array}$} \\
\hline & $\begin{array}{l}\text { Total patients } \\
\text { (incidence) }\end{array}$ & $\begin{array}{l}\text { CBD stones } \\
\text { (incidence) }\end{array}$ & $\begin{array}{l}\text { Total patients } \\
\text { (incidence) }\end{array}$ & $\begin{array}{l}\text { CBD stones } \\
\text { (incidence) }\end{array}$ & & \\
\hline Cholangitis & $19(15.83 \%)$ & $13(68.4 \%)$ & $55(10.45 \%)$ & $32(58.2 \%)$ & 0.179 & 0.59 \\
\hline Total bilirubin $>4 \mathrm{mg} / \mathrm{dL}$ & $34(28.3 \%)$ & $25(73.5 \%)$ & $46(8.74 \%)$ & $38(82.6 \%)$ & $<0.0001$ & 0.41 \\
\hline Total bilirubin $1.8-4 \mathrm{mg} / \mathrm{dL}$ & $31(25.83 \%)$ & $15(48.4 \%)$ & $100(19.01 \%)$ & $64(64.0 \%)$ & 0.09 & 0.14 \\
\hline Biliary pancreatitis & $29(24.16 \%)$ & $15(51.7 \%)$ & $132(25.09)$ & $64(48.5 \%)$ & 0.907 & 0.84 \\
\hline Total bilirubin $<1.8 \mathrm{mg} / \mathrm{dL}$ & $55(45.83 \%)$ & $17(30.9 \%)$ & $380(72.24 \%)$ & $139(36.6 \%)$ & $<0.001$ & 0.45 \\
\hline CBD size on $U S>6 \mathrm{~mm}$ & $30(25 \%)$ & $18(60.0 \%)$ & $280(53.23 \%)$ & $113(40.4 \%)$ & $<0.001$ & 0.051 \\
\hline Abnormal LFT & $72(60 \%)$ & $44(61.1 \%)$ & $414(78.7 \%)$ & $207(50 \%)$ & 0.00018 & 0.09 \\
\hline
\end{tabular}

ASGE, American Society of Gastrointestinal Endoscopy; CBD, common bile duct; LFT, liver function test; US, ultrasound examination 
among females, all risk factors lacked a statistically significant association. Male patients with abnormal LFTs had significantly lower odds (odds ratio [OR] 0.33, 95\% confidence interval $[C I]=0.131-0.827, P=0.02$ ) for choledocholithiasis, whereas female patients with a CBD size $>6 \mathrm{~mm}$ had significantly higher odds ( $\mathrm{OR} 1.9,95 \% \mathrm{CI}=1.135-3.048, \mathrm{P}=0.01$ ) for choledocholithiasis. These parameters were further subjected to receiver operator curve analysis to evaluate their accuracy in determining choledocholithiasis in the two sexes. Among all the parameters, CBD size on US was found to have a significant area under the curve (area under the curve 0.786; 95\%CI 0.644-0.927; $\mathrm{P}=0.006$ ). A cutoff of $4.5 \mathrm{~mm}$ for CBD size on US would have $65 \%$ sensitivity and $78 \%$ specificity, compared to $35 \%$ sensitivity and nearly $100 \%$ specificity for a $6 \mathrm{~mm}$ cutoff.

The patients included in the study were divided into high, intermediate and low likelihood for choledocholithiasis based on the ASGE criteria (Table 3). Of composite study population, $40.0 \%$ males and $24.5 \%$ females were categorized as high risk. In the high-likelihood category, $70.8 \%$ of males and $72.1 \%$ of females had CBD stones, reflecting the high accuracy of the ASGE criteria in predicting CBD stones for this category $(\mathrm{P}=0.85)$. The majority of males $(56.7 \%)$ and females $(70.2 \%)$ were categorized as having intermediate risk $(\mathrm{P}=0.0025)$. In the intermediate-likelihood category, $33.8 \%$ of male and $39.6 \%$ of female patients had CBD stones $(\mathrm{P}=0.42)$. A very small fraction of the study group was categorized as low likelihood for both males (3.3\%) and females (5.3\%); $0 \%$ of males and $14.3 \%$ of females in this category were found to have CBD stones $(\mathrm{P}=0.99)$.

\section{Discussion}

Choledocholithiasis is a common problem in the USA and other western cultures. The burden of disease secondary to stones in the CBD is significant. Significant morbidity and mortality is associated with complications of CBD stones, including conditions such as cholangitis and biliary pancreatitis. Several studies have questioned the real world utility of the ASGE criteria in predicting choledocholithiasis $[10,11]$. Female sex is a known risk factor for gallstone disease, but the ASGE risk criteria for predicting the likelihood of choledocholithiasis do not take sex into consideration. Our cohort study aimed to determine any sex-related differences in the prediction of choledocholithiasis using the ASGE risk criteria.
The composite incidence of choledocholithiasis for males (47.5\%) and females (46.2\%) differed only minimally. The incidence of choledocholithiasis was higher for female patients than for males across the low (14.3\% vs. $0 \%)$, intermediate (39.6\% vs. $33.8 \%)$ and high $(72.1 \%$ vs. $70.8 \%)$ risk ASGE categories $(\mathrm{P}>0.05)$. Five out of eight ASGE risk factors among males, in contrast to four of eight among females, were individually associated with a $>50 \%$ incidence of choledocholithiasis. Total bilirubin $>4 \mathrm{mg} / \mathrm{dL}$, cholangitis, abnormal LFTs (transaminitis), CBD dilation $>6 \mathrm{~mm}$ on US, and biliary pancreatitis were individually associated with choledocholithiasis in $73.5 \%, 68.4 \%, 61.1 \%, 60.0 \%$, and $51.7 \%$ of males, respectively. The association was not in the same order for the female cohort. Total bilirubin $>4$ and $1.8-4 \mathrm{mg} / \mathrm{dL}$, cholangitis, and transaminitis were individually associated with choledocholithiasis in $82.6 \%, 64.0 \%, 58.2 \%$, and $50.0 \%$ of females, respectively. No statistically significant difference between male and female patients was observed in the incidence of choledocholithiasis across individual ASGE-defined risk factors. Despite the lack of statistical significance, these trends suggest there is a need to re-evaluate the strength of association of individual risk factor for choledocholithiasis separately for males and females. A study by Suarez et al showed that a second set of laboratory tests failed to improve the diagnostic accuracy of ASGE criteria in predicting choledocholithiasis [10]. In addition, the authors also concluded that even a significant decline in LFTs did not reliably predict spontaneous stone passage [10].

Further stratification based on the ASGE risk factors for choledocholithiasis revealed an interesting distribution pattern across the low-, intermediate- and high-likelihood categories for each sex. The majority of male (56.7\%) and female (70.2\%) patients with suspected CBD stones were categorized by the ASGE criteria as having intermediate likelihood. More than one third of these patients (33.8\% of males, $39.8 \%$ of females) were eventually found to have CBD stones. This casts doubt upon the true utility of these criteria in helping physicians decide the best next step in the clinical workup of these patients, potentially delaying therapy and increasing the risk of complications in this, the largest subgroup. More than $70 \%$ of subjects (either sex) in the high-likelihood group were found to have CBD stones, clearly demonstrating the high specificity of the ASGE criteria for this subgroup. Interestingly, these criteria showed a high negative predictive value for CBD stones among male patients classified as low likelihood, but failed to do the same for the respective female cohort. A small but significant

$\underline{\text { Table } 3 \text { Incidence of CBD stones across ASGE risk categories (for choledocholithiasis) in male and female patients }}$

\begin{tabular}{|c|c|c|c|c|c|}
\hline \multirow[t]{2}{*}{ ASGE risk strata } & \multicolumn{2}{|c|}{ Male patients } & \multicolumn{2}{|c|}{ Female patients } & \multirow{2}{*}{$\begin{array}{c}\text { P-value } \\
\text { for } \mathrm{CBD} \text { stone incidence }\end{array}$} \\
\hline & $\begin{array}{l}\text { Total patients } \\
\qquad(\mathrm{n}=120)\end{array}$ & $\begin{array}{l}\text { CBD stone incidence } \\
\qquad(47.5 \%)\end{array}$ & $\begin{array}{l}\text { Total patients } \\
\qquad(\mathrm{n}=526)\end{array}$ & $\begin{array}{l}\text { CBD stone incidence } \\
\qquad(46.2 \%)\end{array}$ & \\
\hline Low likelihood & $4(3.3 \%)$ & 0 & $28(5.3 \%)$ & $4(14.3 \%)$ & 0.99 \\
\hline Intermediate likelihood & $68(56.7 \%)$ & $23(33.8 \%)$ & $369(70.2 \%)$ & $146(39.6 \%)$ & 0.42 \\
\hline High likelihood & $48(40.0 \%)$ & $34(70.8 \%)$ & $129(24.5 \%)$ & $93(72.1 \%)$ & 0.85 \\
\hline
\end{tabular}

ASGE, American Society of Gastrointestinal Endoscopy; CBD, common bile duct 


\section{Summary Box}

\section{What is already known:}

- Gallstone disease is a widespread disorder

- Female sex is one of the major risk factors for gallstone disease

- American Society of Gastrointestinal Endoscopy (ASGE) criteria are widely used to risk stratify patients with symptomatic cholelithiasis for concomitant choledocholithiasis

- Current ASGE criteria do not take sex into account in predicting the risk of choledocholithiasis

\section{What the new findings are:}

- The incidence of choledocholithiasis was higher for female patients than for male patients across the low-, intermediate- and high-likelihood ASGE categories

- The ASGE criteria stratify the majority of patients as intermediate risk, with more than one third found to have choledocholithiasis

- The ASGE criteria showed a high negative predictive value for choledocholithiasis among the low-risk category for males, but not for females

percentage $(14.8 \%)$ of females in the low-likelihood group were found to have $\mathrm{CBD}$ stones, which again puts these criteria in question.

Our study had some limitations. Its retrospective nature limited our ability to extract information regarding length of hospital stay, or additional data from modalities such as endoscopic US or magnetic resonance cholangiopancreatography. Despite the large sample size, the trends we observed failed to reach statistical significance. In our study, the observed incidence of CBD stones was similar for both sexes. However, a sex-related difference in the association of clinical, radiological and laboratory risk factors with the risk of choledocholithiasis was noted. The current ASGE criteria, which do not take sex into consideration, predominantly stratifies both male and female patients as having intermediate risk, which in the majority of cases fails to help the physician manage these patients in the real world. Larger prospective studies are needed to improve the current ASGE criteria so as to achieve better risk stratification of patients with suspected choledocholithiasis, improve patient outcomes, and reduce the associated healthcare costs.

\section{References}

1. Everhart JE, Khare M, Hill M, Maurer KR. Prevalence and ethnic differences in gallbladder disease in the United States. Gastroenterology 1999;117:632-639.

2. Völzke H, Baumeister SE, Alte D, et al. Independent risk factors for gallstone formation in a region with high cholelithiasis prevalence. Digestion 2005;71:97-105.

3. Diehl AK. Epidemiology and natural history of gallstone disease. Gastroenterol Clin North Am 1991;20:1-19.

4. Jørgensen T. Gallstones and plasma lipids in a Danish population. Scand J Gastroenterol 1989;24:916-922.

5. Collins C, Maguire D, Ireland A, Fitzgerald E, O'Sullivan GC. A prospective study of common bile duct calculi in patients undergoing laparoscopic cholecystectomy: natural history of choledocholithiasis revisited. Ann Surg 2004;239:28-33.

6. Prat F, Meduri B, Ducot B, Chiche R, Salimbeni-Bartolini R, Pelletier G. Prediction of common bile duct stones by noninvasive tests. Ann Surg 1999;229:362-368.

7. O'Neill CJ, Gillies DM, Gani JS. Choledocholithiasis: overdiagnosed endoscopically and undertreated laparoscopically. ANZ J Surg 2008;78:487-491.

8. Petelin JB. Laparoscopic common bile duct exploration. Surg Endosc 2003;17:1705-1715.

9. Maple JT, Ben-Menachem T, Anderson MA, et al; ASGE Standards of Practice Committee. The role of endoscopy in the evaluation of suspected choledocholithiasis. Gastrointest Endosc 2010;71:1-9.

10. Nárvaez Rivera RM, González González JA, Monreal Robles R, et al. Accuracy of ASGE criteria for the prediction of choledocholithiasis. Rev Esp Enferm Dig 2016;108:309-314.

11. Suarez AL, LaBarre NT, Cotton PB, Payne KM, Coté GA, Elmunzer BJ. An assessment of existing risk stratification guidelines for the evaluation of patients with suspected choledocholithiasis. Surg Endosc 2016;30:4613-4618. 
6 A. Chhoda et al

\section{Supplement Tables}

Supplemental Table 1 Logistic univariate analysis of ASGE risk factors for choledocholithiasis among male patients

\begin{tabular}{lccccc} 
Parameters & Coefficient & Standard error & P-value & Odds ratio & 95\% confidence interval \\
\hline Age $>55$ years & -0.485 & 0.763 & 0.525 & 0.616 & $0.138-2.749$ \\
Total bilirubin & 0.208 & 0.185 & 0.261 & 1.231 & $0.857-1.767$ \\
Abnormal LFT & 1.113 & 0.471 & 0.018 & 0.329 & $0.131-0.827$ \\
Biliary pancreatitis & 0.098 & 0.505 & 0.846 & 1.103 & $0.410-2.970$ \\
CBD size on US & 0.089 & 0.068 & 0.192 & 1.093 & $0.956-1.250$ \\
$>6$ mm & & & & 0.459 & $0.170-1.244$ \\
Cholangitis & -0.778 & 0.508 & 0.126 & 0.45 \\
\hline
\end{tabular}

ASGE, American Society of Gastrointestinal Endoscopy; CBD, common bile duct; LFT, liver function test

Supplemental Table 2 Logistic univariate analysis of ASGE risk factors for choledocholithiasis among female patients

\begin{tabular}{|c|c|c|c|c|c|}
\hline Parameters & Coefficient & Standard error & P-value & Odds ratio & $95 \%$ confidence interval \\
\hline Age $>55$ years & 0.968 & 0.726 & 0.183 & 2.632 & $0.634-10.927$ \\
\hline Abnormal LFT & -0.926 & 0.910 & 0.309 & 0.396 & $0.067-2.359$ \\
\hline Biliary pancreatitis & -3.93 & 0.768 & 0.618 & 0.682 & $0.151-3.070$ \\
\hline $\begin{array}{l}\text { CBD size on US } \\
>6 \mathrm{~mm}\end{array}$ & 0.621 & 0.252 & 0.014 & 1.860 & $1.135-3.048$ \\
\hline Cholangitis & 0.912 & 1.103 & 0.409 & 2.4889 & $0.286-21.66$ \\
\hline
\end{tabular}

ASGE, American Society of Gastrointestinal Endoscopy; CBD, common bile duct; LFT, liver function test 